\title{
Bioelectric Phenomena Accompanying Osmosis in a Single Plant Cell
}

\author{
Yuichiro Nishizaki ${ }^{2}$ \\ Department of Biology, Faculty of Science, Osaka University, Osaka
}

Received June 7, 1954

When one half of an internodial cell of Nitella is covered with water and the other half is in contact with the solution of sucrose, water enters at the former, flows along the inside of the cell, and escapes at the latter due to the difference in so-called suction forces, or in "diffusion pressure deficits", a terminology familiar to the American workers, at the two halves of the cell (Osterhout, 1949 a; Kamiya, Tazawa and Kuroda, 1952).

Since solutes in the cell sap as well as in the external solutions hardly go out of or into the cell, transport of water from one end of the cell to the other brings about the intracellular concentration gradient which was demonstrated by Kamiya and Kuroda (1953) in a quantitative manner. This concentration gradient of the sap is accompanied by a difference in the electric potential between the two ends of one and the same cell. The present work was undertaken to find how the potential difference (P.D.) thus elicited changes with time when the two halves of the cell are submerged in the two solutions whose osmotic pressures are different from each other due to the uneven concentration of the nonelectrolyte.

\section{Material and methods}

Observations were made on the internodial cell of Nitella flexilis ${ }^{3}$. The cells, 4-5 cm in length, were freed from neighbouring cells and immersed in advance in a $\mathrm{KCl}$ solution at the same concentration as that to be used for the experiment for at least one hour.

In the experiments, the internodial cell was placed in a paraffin vessel consisting of two pools in such a way as one half of a cell (Fig. 1, $a$ ) is placed in pool $A$ and the other half (Fig. 1, $b$ ) is placed in another pool $B$. The cell was mounted at the middle region in a narrow groove in the septum with a small amount of vaseline which helps to make a water tight seal. A pair of calomel electrodes, $\mathrm{E}_{A}$ and $\mathrm{E}_{B}$, was connected through the salt-agar bridges with the solutions oft wo pools, $A$ a nd $B$. The salt-agar bridges contained $\mathrm{KCl}$ solution having the same concentration as that of the solutions

1 This work has been supported by a Grant for Fundamental Scientific Research from the Ministry of Education. The results of the experiments presented here were reported briefly at the XVIIth Annual Meeting of the Botanical Society of Japan (1952).

2 Present address: Institute for Agricultural Research, Tôhoku University, Sendai.

3 The material was supplied from the Botanical Garden of Kyoto University. 
in pools $A$ and $B$. The two calomel electrodes were led to the potentiometer through the current reverser. Experiments were performed at $28^{\circ} \mathrm{C}$.

At first the $\mathrm{KCl}$ solution in a definite concentration, e.g., $0.05 \mathrm{M} \mathrm{KCl}$, was admitted to both pools $A$ and $B$. The P.D. between the two pools was then approximately null. If, however, the $\mathrm{KCl}$ solution in pool $B$ is replaced with the same $\mathrm{KCl}$ solution to which sucrose is added so that the final sucrose concentration amounts to $0.2 \mathrm{M}$, while $\mathrm{KCl}$ solution in pool $A$ remained the same, the osmotic pressure in $B$ becomes higher than that of the solution in $A$ by $0.2 M$. Water therefore goes into the cell at pool $A$, passes along the inside of the cell and goes out of the cell at pool $B$. Under such circumstances the P.D. between the two pools, $A$ and $B$, is no longer kept at zero, but shows pronounced variations, although the concentration of $\mathrm{KCl}$ in the two pools remains

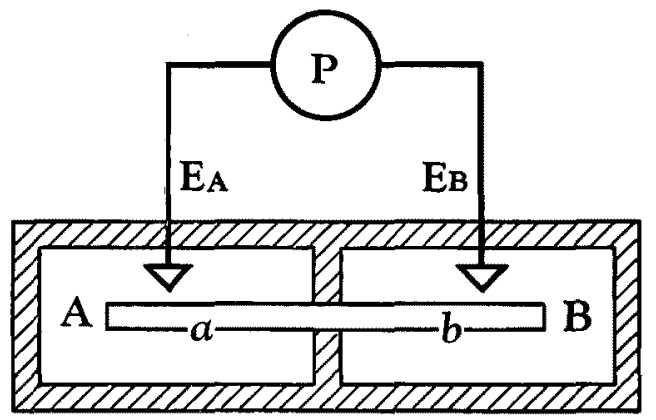
practically the same, as the transport volume of water through the cell is negligibly small as compared with the amount of solutions in the two pools. The time course of changes in P.D. induced by osmosis is, however, different according to the concentration of $\mathrm{KCl}$ in the external media under the same osmotic gradient induced by sucrose. The concentrations of $\mathrm{KCl}$ so far used

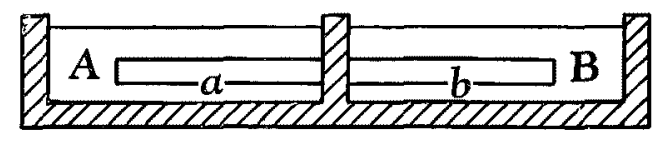

Fig. 1. Diagram of the vessel consisting of two pools $A$ and $B$ (top: general view, bottom: side view). Two parts of a Nitella cell in pools $A$ and $B$ are designated as $a$ and $b$ respectively. $\mathrm{E}_{A}$ and $\mathrm{E}_{B}$ : non-polarizable electrodes. P: potentiometer. were $0.05 M, 0.01 M, 0.001 M$, and $0.0001 M$.

When the solution in pool $B$, such as $0.05 \mathrm{M} \mathrm{KCl}+0.2 M$ sucrose, is replaced after a while with the original solution without sucrose, such as 0.05 $M \mathrm{KCl}$, water begins to flow in the reverse direction through the cell entering it at $b$ end and leaving it at $a$ end as already pointed out by Osterhout (1949a) and Kamiya et. al. (1952). The P.D. between $A$ and $B$ also begins to go back at this time to the original value which was null. If this value did not return to zero or if the protoplasmic streaming was found to be abnormal, the experiment was rejected.

\section{Results}

1) $0.05 \mathrm{M}-0.01 \mathrm{M} \mathrm{KCl}$ Solutions

The changes in P.D. accompanying osmosis when $\mathrm{KCl}$ concentration of 
the media was $0.05 M$ are shown in Fig. 2. At the start when the two halves of the cell, $a$ and $b$, were covered both with $0.05 M \mathrm{KCl}$ solution, there appeared scarcely any P.D. between them (I in Fig. 2). Once, however, the solution of $0.05 \mathrm{M} \mathrm{KCl}+0.2 M$ sucrose was exchanged for the solution of $0.05 M \mathrm{KCl}$ in $B$, the potential at $B$ against $A$ began to increase immediately (II in Fig. 2). After about 4 minutes the potential reaches a steady value amounting to approximately $17 \mathrm{mV}$. By replacing the solution $(0.05 M \mathrm{KCl}$ $+0.2 M$ sucrose) in $B$ with $0.05 \mathrm{M} \mathrm{KCl}$ again, we saw that the induced P.D. soon began to decrease arriving eventually at null (III in Fig. 2).

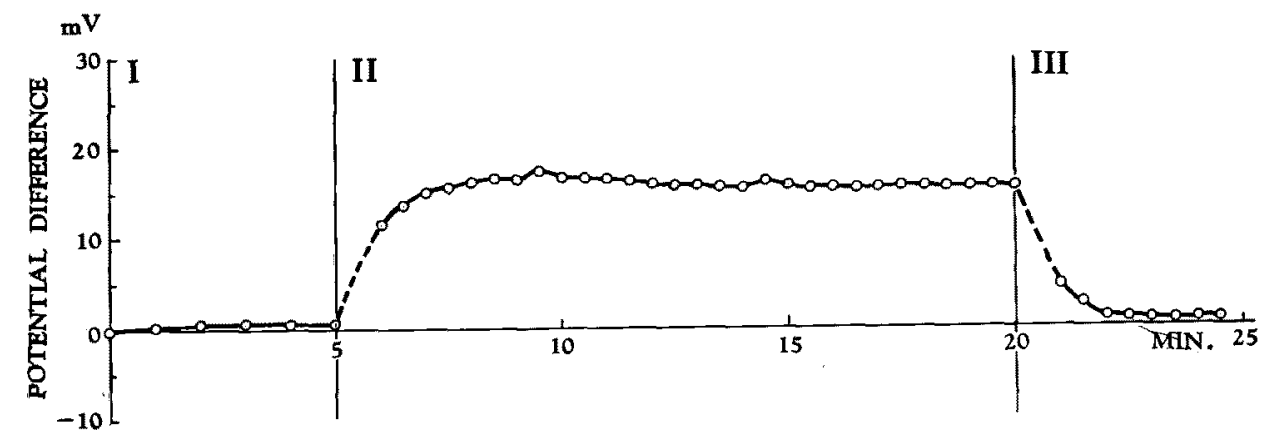

Fig. 2. Changes in the electric potential at $B$ against $A$. $I: 0.05 M \mathrm{KCl}$ in $A$ and $B$. II: $0.05 M \mathrm{KCl}$ in $A$ and $0.05 M \mathrm{KCl}+0.2 M$ sucrose in $B$. III: $0.05 M \mathrm{KCl}$ in $A$ and $B$.

The above experiment shows that when the concentration of $\mathrm{KCl}$ in the external media of the cell is $0.05 \mathrm{M}$, the pool where water comes out of the cell becomes higher in electric potential than the pool where water goes into the cell. Nearly the same feature of changes was observed also when the concentration of $\mathrm{KCl}$ was $0.01 \mathrm{M}$.

In the above experiment it may be reasonably admitted that sucrose, being a non-electrolyte, does not affect the bioelectrical potential of a cell directly. On the other hand, the two parts of the cell being always in contact with the same concentration of $\mathrm{KCl}, \mathrm{KCl}$ existing in the external media must not be responsible for the observed P.D. Therefore it must be assumed that the P.D. is established in some connection with the transport of water through the cell due to the difference in the osmotic pressures in the external solutions.

It has been reported that the movement of water from $A$ to $B$ through the cell carries solutes in the vacuole from $a$ to $b$ but solutes cannot pass the protoplasmic layer except very slowly and consequently they become more diluted at the left half $a$ and more concentrated at the right half $b$ of the cell.

It was shown by Osterhout (1930 etc.) that the P.D. between the inside and outside of Nitella cell is largely due to $\mathrm{K}^{+}$having a much larger mobility than $\mathrm{Cl}^{-}$in the protoplasmic layer ${ }^{2}$ and that it changes in such a fashion as

1. The protoplasmic layer, which actually consists of the protoplasmic membrane, resting plasmagel layer, flowing plasmasol layer and vacuoler membrane (tonoplast), is regarded here as a single membrane for the sake of simplicity. 
to obey the equation of Nernst and Henderson for diffusion potential.

According to the equation of Nernst and Henderson for diffusion potential, the P.D.'s between the inside and outside of the cell at $a$ and $b$ are

$$
\begin{aligned}
& \mathrm{E}_{x}=\frac{\mathrm{RT}}{\mathrm{F}} \mathrm{U}_{\mathrm{K}}-\mathrm{V}_{\mathrm{Cl}} \ln \frac{\mathrm{C}_{a}}{\mathrm{U}_{\mathrm{K}}+\mathrm{V}_{\mathrm{Cl}}}, \\
& \mathrm{E}_{b}=\frac{\mathrm{RT}}{\mathrm{F}} \frac{\mathrm{U}_{\mathrm{K}}-\mathrm{V}_{\mathrm{Cl}}}{\mathrm{U}_{\mathrm{K}}+\mathrm{V}_{\mathrm{C}_{1}}} \ln \frac{\mathrm{C}_{b}}{\mathrm{C}_{B}},
\end{aligned}
$$

where $F, R$ and $T$ are the Faraday constant, gas constant and absolute temperature respectively; $\mathrm{U}_{\mathrm{K}}$ and $\mathrm{V}_{\mathrm{Cl}}$ are the relative mobilities of $\mathrm{K}^{+}$and $\mathrm{Cl}^{-}$ in the protoplasmic layer and actually $\mathrm{V}_{\mathrm{Cl}}$ is taken as unity; $\mathrm{C}_{a}$ and $\mathrm{C}_{b}$ are concentrations $^{2}$ of $\mathrm{KCl}$ in the cell sap at $a$ and $b$; and $\mathrm{C}_{A}$ and $\mathrm{B}_{B}$ are the concentrations of $\mathrm{KCl}$ in the solutions in pools $A$ and $B$ respectively. In this experiment, the potential at $B$ against $A$, or $\mathrm{E}_{b}-\mathrm{E}_{\lrcorner}$was measured. Consequently if we assume that $C_{A}$ was kept equal to $C_{B}$ during the experiment, we get the following relation from equations (1) and (2):

$$
\mathrm{E}_{b}-\mathrm{E}_{a}=\frac{\mathrm{RT}}{\mathrm{F}}-\frac{\mathrm{U}_{\mathrm{K}}-\mathrm{V}_{\mathrm{Cl}}}{\mathrm{U}_{\mathrm{K}}+\mathrm{V}_{\mathrm{Cl}}} \ln \frac{\mathrm{C}_{b}}{\mathrm{C}_{a}} .
$$

As $\frac{U_{K}-V_{O_{1}}}{U_{K}+V_{C_{1}}}$ is known to be nearly equal to $1^{3}$ under the normal conditions of the cell, we get $\mathrm{E}_{b}-\mathrm{E}_{a}>0$, if $\mathrm{C}_{b}>\mathrm{C}_{u}$. This is indeed the case in Fig. 2. According to the experiment performed by Kamiya and Kuroda (1953) the osmotic pressure of the cell sap in $a$ reached a level as low as $0.18 \mathrm{M}$ and that in $b$ went as high as $0.34 \mathrm{~mol}$ in 15 minutes after the osmotic pressure of the medium in $B$ was made higher than that in $A$ by $0.2 \mathrm{M}$. Under such circumstances, electrolytes contained in the cell sap must also be diluted at $a$ and concentrated at $b$. The normal osmotic pressure of the cell sap being $0.26 M$ of which the $\mathrm{KCl}$ concentration is about $0.1 M, \mathrm{KCl}$ concentrations in $a$ and $b$ must be $0.13 M$ and $0.07 M$ respectively in the above situation. Thus, assuming the value of $\mathrm{U}_{\mathrm{K}} / \mathrm{V}_{\mathrm{C} 1}$ to be 73 after Osterhout, we are in a position to calculate from equation (3) the P.D. between $A$ and $B$ to be expected on the basis of diffusion potential. Namely, we get

$$
\mathrm{E}_{b}-\mathrm{E}_{a}=60 \frac{73-1}{73+1} \log \frac{0.13}{0.07}=16 \mathrm{mV} .
$$

This value nearly coincides with the observed value of P.D. which was $17 \mathrm{mV}$ after the P.D. attained equilibrium. Therefore the value of 73 for $U_{K} / V_{\mathrm{Cl}}$ obtained by Osterhout applies in this case.

2 Exactly speaking, they represent the activities, but for practical purposes we can substitute concentrations for them.

3 According to Osterhout (1930) $\mathrm{U}_{\mathrm{K}}: \mathrm{V}_{\mathrm{CI}}=73: 1$

$4 \mathrm{~K}^{+}$concentration in the cell sap of Nitella flexilis used for the present work was found by means of the flame-photometric analysis to be between $0.087-0.110 M$ (Kamiya and Kuroda, unpublished). 


\section{2) $0.001 M-0.0001 M$ Solutions}

On the other hand, when the concentration of $\mathrm{KCl}$ in the two pools was once reduced to $0.001 M$ while the osmotic gradient to which the cell was subjected remained the same, the P.D. between $A$ and $B$ follows a time course (Fig. 3) different from that observed in $0.05 M-0.01 M \mathrm{KCl}$ solutions. By replacing $0.001 \mathrm{M} \mathrm{KCl}$ solution in pool $B$ with the solution of



Fig. 3. Changes in the electric potential at $B$ against $A$. I: $0.001 M \mathrm{KCl}$ in $A$ and $B$. II: $0.001 M \mathrm{KCl}$ in $A$ and $0.001 M \mathrm{KCl}+0.2 M$ sucrose in $B$. III $0.001 M \mathrm{KCl}$ in $A$ and $B$.

$0.001 \mathrm{M} \mathrm{KCl}+0.2 M$ sucrose while $0.001 M \mathrm{KCl}$ in pool $A$ remained unchanged, we observed that the potential at $B$ against $A$ once became positive, but it soon began to decrease rapidly until it reached around $-65 \mathrm{mV}$ (II in Fig. 3). It is noticeable in this case that the curve of P.D. showed some irregular deflections indicating the unstable state of the cell which was not the case in $0.05 \mathrm{M} \mathrm{KCl}$. If $0.001 \mathrm{M} \mathrm{KCl}$ was again substituted for the solution of $0.001 \mathrm{MKCl}+0.2 \mathrm{M}$ sucrose in pool $B$, the diminished potential at $B$ against $A$ gradually came back to the initial state (III in Fig. 3).

A further unexpected feature of the behaviour of the cell was observed in the solution of $0.0001 M \mathrm{KCl}$. As soon as the $0.0001 \mathrm{M} \mathrm{KCl}+0.2 \mathrm{M}$ sucrose solution was admitted to $B$ while $A$ is filled with $0.0001 M \mathrm{KCl}$, the curve of the P.D. takes a downward trend from the beginning showing some irregular deflections (II in Fig 4). When pure $0.0001 \mathrm{M} \mathrm{KCl}$ solution was readmitted to $B$ in place of $0.0001 M \mathrm{KCl}+0.2 M$ sucrose, the P.D. between $A$ and $B$ gradually diminished until the initial condition is restored (III in Fig. 4). The form of the curve represented in III in Fig. 4 is quite similar to that shown in Fig. 3.

Equation (3) shows that the values of P.D. to be observed, $E_{b}-E_{a}$, are the function not of the absolute concentrations of external $\mathrm{KCl}$ solutions, but of the ratio of concentrations of $\mathrm{KCl}$ in the cell sap at both ends. Therefore, so far as the concentration of sucrose in $B$ is the same as that in experiment (1), it is expected that the potential at $B$ in referenec to $A$ would be positive 
and would follow a time course similar to the curve shown in Fig. 2 independently of the concentrations of the external $\mathrm{KCl}$ solutions. The above experiments show, however, that when the concentrations of $\mathrm{KCl}$ in the outer media are decreased to $0.001 M-0.0001 M$, the P.D. follows a time course other than that observed in $0.05 \mathrm{M} \mathrm{KCl}$.

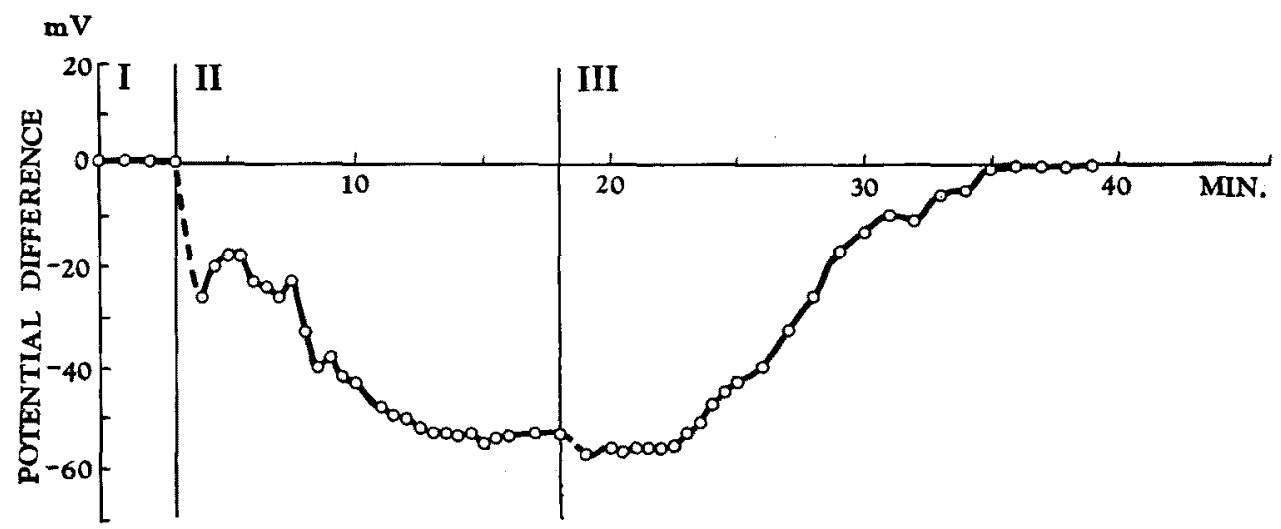

Fig. 4. Changes in the electric potential at $B$ against $A$. I: $0.0001 M \mathrm{KCl}$ in $A$ and $B$. II: $0.0001 M \mathrm{KCl}$ in $A$ and $0.0001 M \mathrm{KCl}+0.2 M$ suerose in $B$. IIr: $0.0001 M \mathrm{KCl}$ in $A$ and $B$.

We supposed previously that the concentration of $\mathrm{KCl}$ in the proximity of the cell surface remained practically constant during the experiment. This presumption is supported by the fact that the stirring of the solution in pools $A$ and $B$ exerted no observable influence on the P.D. measured. A further support for this is to be obtained by experiments in which fresh solutions are substituted, after the P.D. curve attained the stationary state, for the solutions in $A$ and $B$ which were originally the same as the substituted ones. By this procedure the potential at $B$ against $A$ showed a temporal, slight change in the direction of increasing its negativity. This brief change in the potential was restored to the value approximately the same as that before exchanging the solutions. These facts seem to indicate that the increase in $\mathrm{KCl}$ concentration in the proximity just outside the cell surface at $B$, which might be expected as a result of possible leakage of electrolytes accompanying osmosis (Kamiya and Kuroda, 1953), is not responsible for the peculiar changes in P.D. shown in Figs. 3 and 4.

A plausible assumption accounting for the different mode of change in P.D. observed when the external $\mathrm{KCl}$ concentration is very low is that the mobility of $\mathrm{K}^{+}$and $\mathrm{Cl}^{-}$would no longer be kept at the normal value but would be varied by the transport of water through the cell. If $\mathrm{E}_{a}$ would increase through the increare of $U_{K}$ on $A$ side or if $E_{b}$ falls through the decrease of $\mathrm{U}_{\mathrm{K}}$ on $B$ side, $\mathrm{E}_{b}-\mathrm{E}_{a}$ may get negative as is the case in Figs. 3 and 4. However, $U_{K}$ is so much largerthan $V_{C l}$ that the increase of $U_{K}$ cannot bring the noticeable increase of $E_{\lrcorner}$. On the contrary if $U_{\mathrm{K}}$ approaches 
$\mathrm{V}_{\mathrm{Cl}}$ in $b, \mathrm{E}_{b}$ decreases as $\frac{\mathrm{U}_{\mathrm{K}}-\mathrm{V}_{\mathrm{Cl}}}{\mathrm{U}_{\mathrm{K}}+\mathrm{V}_{\mathrm{Cl}}}$ decreases. Thus it is probable that the decrease of the relative mobility of $\mathrm{K}^{+}$in $b$ where water comes out from the cell is responsible for the negative potential at $B$ against $A$.

\section{3) Direct Measurements}

Direct proof as to the decrease of $\mathrm{E}_{0}$, when the $\mathrm{KCl}$ concentration is low, is to be obtained by the experiment using a microelectrode. By inserting a microelectrode into a vacuole while electrodes $\mathrm{E}_{A}$ and $\mathrm{E}_{B}$ are immersed in pools $A$ and $B$, it is possible to measure both $\mathrm{E}_{a}$ and $\mathrm{E}_{\iota}$ independently. The results of the experiment (Fig. 5) showed that $\mathrm{E}_{\iota}$ decreased only slightly as

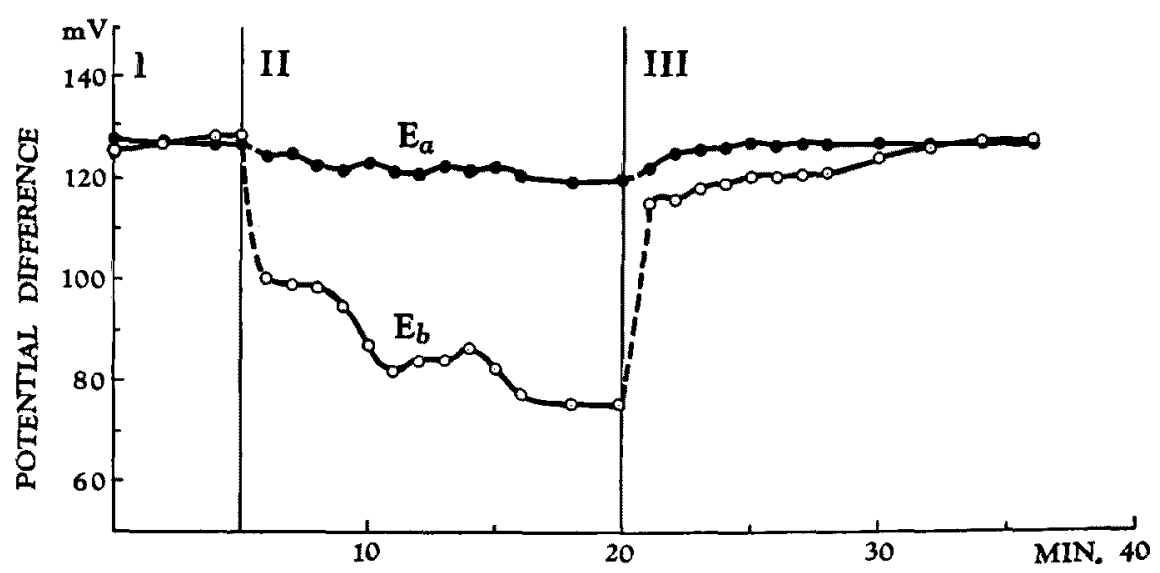

Fig. 5. Changes in the electric potential at the outside of the cell against the inside, as measured by inserting a microelectrode into a vacuole. $\mathrm{E}_{a}:$ P. D. at $A . \mathrm{E}_{b}: \mathrm{P}$. D. at $B$. I: $0.0001 M \mathrm{KCl}$ in $A$ and $B$. II: $0.0001 M \mathrm{KCl}$ in $A$ and $0.0001 M \mathrm{KCl}+0.2 M$ sucrose in $B$. III: $0.0001 M \mathrm{KCl}$ in $A$ and $B$. Temperature: $22^{\circ} \mathrm{C}$.

the ionic concentration in $a$ was diluted, while $\mathrm{E}_{b}$ decreased considerably in spite of the increase in the ion concentration in $b$. As already stated, the $\mathrm{KCl}$ concentration of the cell sap in $b$ will be $0.13 \mathrm{M}$ in 15 minutes after the solution in $B$ has been made higher in osmotic pressure than that in $A$ by $0.2 \mathrm{M}$. Hence, if we might venture to calculate the relative value of $\mathrm{U}_{\mathrm{K}}$ at $b$ from equation (2) by substituting $\mathrm{C}_{b}=0.13 M, \mathrm{C}_{B}=0.0001 M, \mathrm{~T}=$ $273+22^{\circ}$ and $E_{b}=75 \mathrm{mV}$, we would find the value of $\mathrm{U}_{\mathrm{K}}$ under exosmosis to be 2.4. This is very small as compared with the normal value which is reported by Osterhout to be 73 .

\section{Discussion}

In the experiments described above, water is forced to enter the cell across the protoplasmic membrane at one end, to move through the cell and go out of it at the other end to the external medium. Under such circumstances the streaming potential might first come under consideration as a cause tor 
the observed P.D. If this be the case, however, the maximum P.D. should be expected when the velocity of the movement of water through the cell is maximum. Since the relation to time of the volume of water transported through the cell is generally represented by the curve designated as $I$ in Fig. 6 (Kamiya, Tazawa and Kuroda, 1952), the maximum P.D. should appear immediately after replacement of $\mathrm{KCl}$ solution in $B$ with $\mathrm{KCl}+0.2 \mathrm{M}$ sucrose solution. The P.D. should then decrease following the time course corresponding to the curve II which represents the differential coefficient of curve I. However, this has never been the actual case. The P.D.

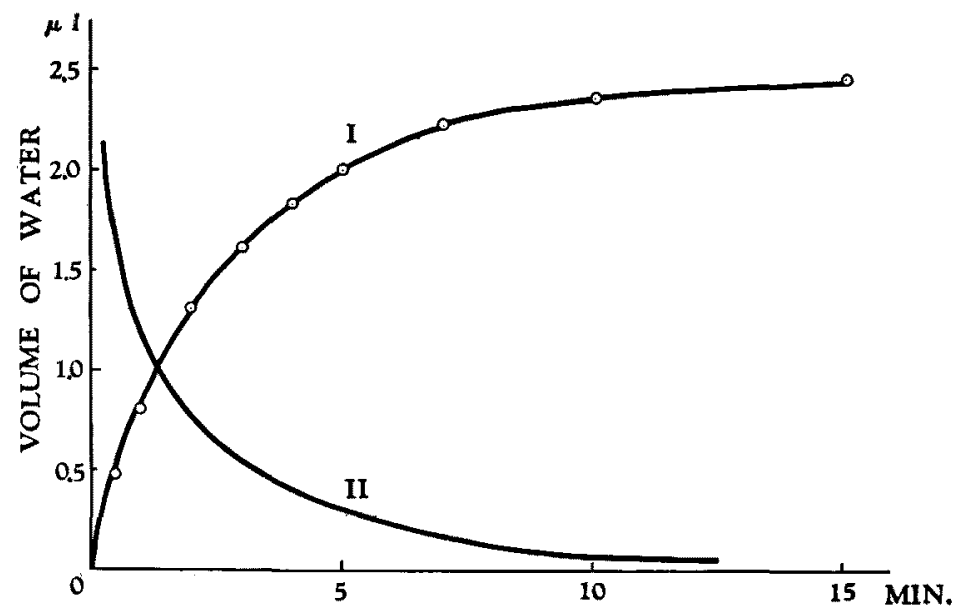
increased with Fig. 6. I: Volume of water transported through the cell under time. It is there- the osmotic drive of $0.2 M$ sucrose (after Kamiya, Tazawa and fore clear that Kuroda, 1952). II: Differential coefficient of curve I.

the streaming

potential does not play an important part for the potential change in osmosis.

Since it has been made clear by Osterhout (1949a), and Kamiya and Kuroda (1953) that the intracellular concentration gradient of the sap is established by the movement of water through the cell, the diffusion potential across the protoplasmic layer is to be taken into account in the above experiments. If the concentration of the extracellular $\mathrm{KCl}$ is comparatively high $(0.05 \mathrm{M}-$ $0.01 \mathrm{M}$ ), the P.D. between inside and outside of the cell changes in accordance with the equation for diffusion potential. However, when extracellular $\mathrm{KCl}$ is lowered to the concentration range of $0.001 M-0.0001 M$. the P.D. between the inside and outside of the cell no longer obeys the equation for diffusion potential unless we assume the changes in the mobility of $\mathrm{K}^{+}$in the protoplasmic layer. This is especially true at the part of the cell where water goes out.

\section{Summary}

1. The potential difference (P.D.) between the two ends of one and the same internodial cell of Nitella was measured when water enters the cell at one end and goes out at the other under the osmotic gradient of $0.2 \mathrm{M}$ sucrose. 
2. When the cell is in contact with the $\mathrm{KCl}$ solution in the concentration range of $0.05 M-0.01 \mathrm{M}$, the potential at the part where water comes out of the cell becomes always higher than that where water enters.

3. This phenomenon is explained by the uneven distribution of $\mathrm{KCl}$ in the cell sap in terms of diffusion potential.

4. When very diluted $\mathrm{KCl}$ solutions $(0.001 M-0.0001 M)$ are applied to the external medium, the P.D. between the two parts of the cell shows discrepancies which are not apprehensible on the basis of diffusion potential unless changes in the mobility of $\mathrm{K}^{+}$in the protoplasmic layer are postulated in the cell part where water flows out.

The author wishes to express his most cordial thanks to Professor N. Kamiya for his helpful advice and directions throughout this work.

\section{References}

Kamiya, N. and Kuroda, K. 1953. XVIIIth Ann. Meeting of Bot. Soc. Japan. Kamiya, N., Tazawa, M. and Kuroda, K, 1952. XVIIth Ann. Meeting of Bot. Soc. Japan. Osterhout, W.J.V. 1930. Caleulations of bioelectric potentials. I. Effects of KCl and $\mathrm{NaCl}$ on Nitella. Jour. Gen. Physiol. 13, 715-732.

- 1949a. Movements of water in cells of Nitella. Jour. Gen. Physiol. 32, 558-557.

- 1949b. Some bioelectrical problems. Proc. Nat. Acad. Sci. 35, 548-558.

P.S. After the present paper was prepared, the recent work of Osterhout entitled "Changes in resting potential due to a shift of electrolytes in the cell produced by non-electrolytes" (Jour. Gen. Physiol. 37. 423-432, 1954) which was carried out along a line similar to but not identical with ours, has arrived in our hands. 